\title{
Ethnobotanical Survey of the Medicinal Plants used in the Southern Mediterranean. Case Study: The Region of Bissa (Northeastern Dahra Mountains, Algeria)
}

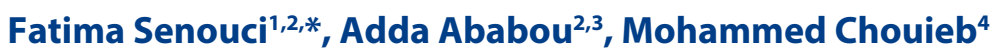

Fatima Senouci ${ }^{1,2, *}$, Adda Ababou $^{2,3}$, Mohammed Chouieb $^{4}$

'Department of Biology, Faculty of Nature and Life Sciences, University Abd El Hamid Ibn Badis, Mostaganem, ALGERIA.

${ }^{2}$ Laboratory of Natural Bioresources, Faculty of Nature and Life Sciences, University Hassiba Ben Bouali, Chlef, ALGERIA. ${ }^{3}$ Department of Biology, Faculty of Nature and Life Sciences, University Hassiba Ben Bouali, Chlef, ALGERIA.

${ }^{4}$ Department of Agronomy, Faculty of Nature and Life Sciences, University Abd EI Hamid Ibn Badis, Mostaganem, ALGERIA.

\section{Correspondence}

Fatima Senouc

Department of Biology, Faculty of Nature and Life Sciences, University Abd El Hamid Ibn Badis, Mostaganem; Laboratory of Natural Bioresources, Faculty of Nature and Life Sciences, University Hassiba Ben

Bouali, Chlef, ALGERIA

Phone no: 00213668025913

E-mail: senoucifatima55@yahoo.fr

History

- Submission Date: 17-01-2019;

- Review completed: 22-04-2019;

- Accepted Date: 31-04-2019.

DOI : 10.5530/pj.2019.11.103

Article Available online http://www.phcogj.com/v11/i4

\section{Copyright}

(C) 2019 Phcogj.Com. This is an openaccess article distributed under the terms of the Creative Commons Attribution 4.0 International license.

\begin{abstract}
Introduction: The present study falls within the framework of valorization of medical plants and traditional knowledge's in the Northeastern part of Dahra Mountain, Algeria. Methods: An ethnobotanical survey was conducted between 2016 and 2018 in order to study the traditional and therapeutic use of medical plants amongst the local population. Data collected was analyzed using quantitative indices such as the fidelity level (FL) and Informant Consensus Factor (FIC). Results: The results revealed 70 medicinal species belonging to 38 botanical families, among which, five families were the most commonly used by the local population, especially the families Lamiaceae and Asteraceae. In term of species, the highest frequencies of medicinal use were shown respectively by Tetraclinis articulata (Vahl) Mast. (6.49\%), Pistacia lentiscus L. (6.11\%), Myrtus communis L. (5.34\%) and Thymus vulgaris L. (4.96\%). Among the various parts of the plant, leaves were the most used part and decoction was the most frequent form of use. Finally, the gastro-intestinal system disorders were the most treated diseases with a percentage of $28.24 \%$. Conclusion: This first ethnobotanical study conducted in the region of Bissa revealed a very rich local knowledge in term of traditional herbal medicine; this fact was reflected by the high diversity of species used in the treatment of several diseases. In light of this, it is therefore very important to subject some of the major species to further phytochemical and pharmacological studies in order to validate their traditional use and to probably discover new bioactive molecules.
\end{abstract}

Key words: Algeria, Ethnobotany, Indigenous population, Medicinal plants, Traditional medicine.

\section{INTRODUCTION}

The study of the traditional use of medicinal plants by indigenous peoples and rural communities belongs to the domain of ethnobotany ${ }^{1,2}$ In most of the developing countries such as Algeria, the knowledge about traditional medicine has continued to be passed orally from generation to generation. ${ }^{3-5}$ Unfortunately, with the recent development of life and the pharmaceutical industry, this knowledge became less and less transmitted and tends to disappear, thus, the necessity to archive and integrate the traditional medicine into the modern health system using ethnobotany and ethnopharmacology. ${ }^{6}$

In this context, several ethnobotanical surveys in relation to traditional medicine have been conducted throughout the world, such as Turkey, ${ }^{7-10}$ Northeastern Brazil, ${ }^{11,12}$ India, ${ }^{13}$ Philippines, ${ }^{14}$ Ghana, ${ }^{15}$ Côte d'Ivoire ${ }^{16}$ and Morocco. ${ }^{17,18}$

Recently, many ethnobotanical and ethnopharmacological studies were undertaken in various regions throughout the Algerian country, in order to preserve the indigenous knowledge and to develop a strategy to protect biodiversity and plant species. ${ }^{4-6,19-28}$ However, due to the large Algerian surface and diversity, most its geographical areas and ethnic communities have still not been explored ethnobotanically. ${ }^{5}$
With the disappearance of the old indigenous connoisseurs, it became very urgent to document the traditional knowledge through ethnobotanical studies, ${ }^{4}$ which is very important for the conservation of this knowledge, the use of the biological resources and at the same time represents an essential tool in the defense of biodiversity. ${ }^{29}$ Therefore, the record of the local names and indigenous uses of plants has significant potential societal benefits. ${ }^{30,31}$ In this context, interviews and inventories represent the most effective way to preserve this knowledge. ${ }^{12}$

The study of the local knowledge on biological resources used in traditional medicine, by rural communities in Zeboudja and Beni Haoua a south Mediterranean districts has become necessary for the valorization and conservation of the local flora in Bissa forest, an area known by its important floristic diversity and where no ethnobotanical surveys were conducted. The main purpose of this study was the elaboration of an ethnobotanical survey in order to identify the medicinal plants and the use of these plants.

\section{MATERIAL AND METHODS}

\section{Study area and population}

The study area, located in the Northwestern side of Algeria in the Eastern part of the Dahra Mountain, is

Cite this article: Senouci F, Ababou A, Chouieb M. Ethnobotanical Survey of the Medicinal Plants used in the Southern Mediterranean. Case Study: The Region of Bissa (Northeastern Dahra Mountains, Algeria). Pharmacog J. 2019;11(4):647-59. 
a coastal ecosystem with a highly-developed forest. It extend between $1^{\circ} 12^{\prime} 56.9^{\prime \prime}$ and $1^{\circ} 42^{\prime} 4.1^{\prime \prime}$ of East longitude and between $36^{\circ} 18^{\prime} 9.5^{\prime \prime}$ and $36^{\circ} 33^{\prime} 26.7^{\prime \prime}$ of North latitude, at $45 \mathrm{~km}$ North of the Chlef state (Figure 1). It belongs to the districts of Zeboudja and Beni Haoua and extends over an area of 36671 ha.

It is a typical Mediterranean area in terms of landscape structure, composition and climate, distinguished by hot, dry summers and relatively rainy winters, with an annual dry period of 5 months and $74 \%$ of the annual precipitations are recorded during winter and autumn. ${ }^{32,33}$

It is a mountainous with elevations ranging from 400 to 1146 meters and woody area dominated by species such as Pinus halepensis L., Quercus suber L., Quercus ilex L., Arbutus unedo L., Erica arborea L. and Cistus monspeliensis L.

In 2017, the total population in the surrounding municipalities of Zebboudja, Beni Houa, Benairia and Berira was 94187 inhabitants, with a male/female ratio of 1.04 . The population is mixed between Arabic and Berber ethnicity and the majority of them are farmers and farm workers. Although the availability of modern health care facilities, this rural population still prefer and practice the traditional medicine.

\section{Ethnobotanical survey}

In this study, the ethnobotany of medicinal plants used in Zeboudja, Beni Haoua and the surrounding areas were investigated over two years, from spring 2016 to spring 2018.

Interviews through a well-structured questionnaire were carried out during the busy hours in the common areas visited by local people such as markets, mosques and cafeterias and also at their homes without time limit. ${ }^{34}$ Through, this questionnaire we recorded information, such as the demographic characteristics of the informant are shown in Table 1 , the local names, the used part, the preparation method and the way of use of the plants.

\section{Plant materials}

A total of 70 species were collected throughout the study area, the local names of these species were given by the local population and the scientific names were provided by botanists and experts using the flora of Quezel and Santa ${ }^{35}$ and the North African database index. ${ }^{36}$

\section{Data analysis}

The ethnobotanical analysis was performed according to several indices (Equation I, II). ${ }^{4-5,13,23,26,37}$

\section{a) Informant consensus factor (FIC)}

This index was given by the following formula:

$F I C=\frac{(N u r-N t)}{(N u r-1)}$

Where Nur is the number of use citations for each ailment category and $\mathrm{Nt}$ is the number of taxa used in that category.

b) Fidelity Level

The Fidelity level (FL) was calculated using the following formula:

$$
F L(\%)=N_{p} / N \times 100
$$

Where Np refers to the number of informants citing the use of the plant for a particular ailment category and $\mathrm{N}$ is the total number of informants citing the plant for any disease category.

\section{RESULTS AND DISCUSSION}

\section{Demographic characteristics of informants}

Overall, this research showed that informants from Berber ethnicity were more used to the traditional medicine and provided us with considerably more information than the informants from Arabic ethnicity.

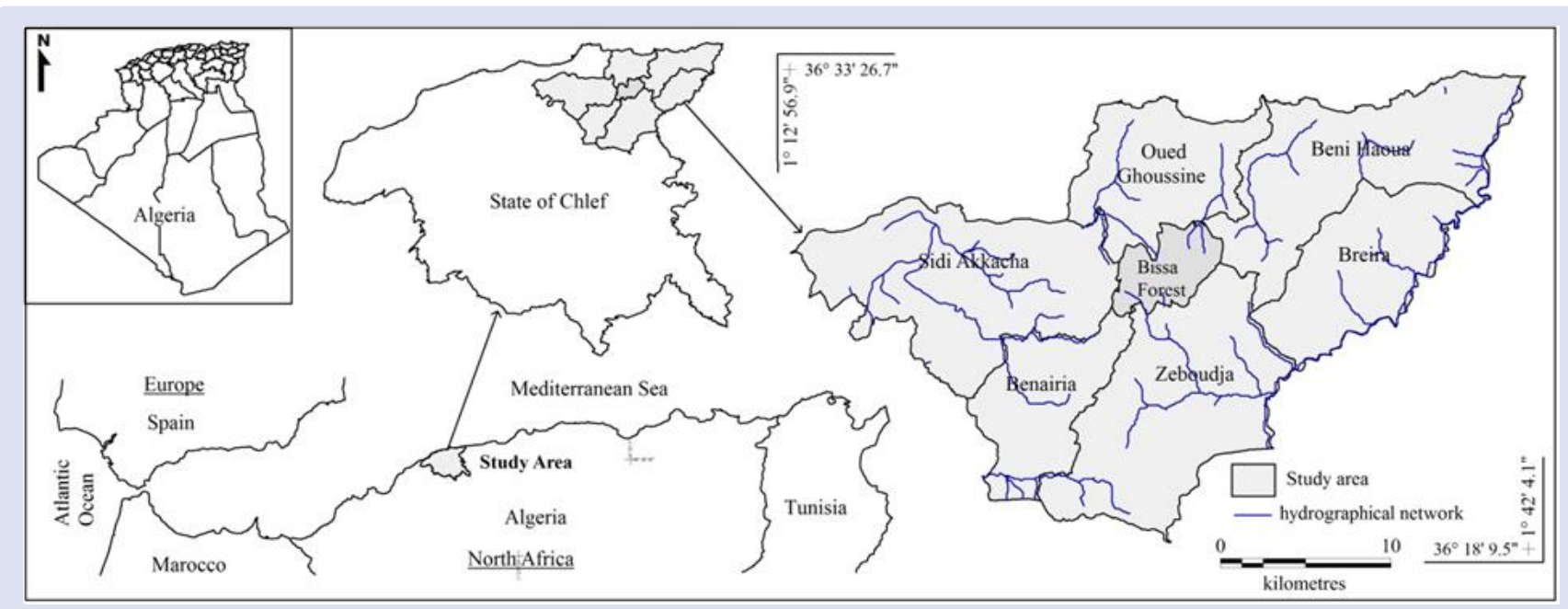

Figure 1: Location of the study area.

Table 1: Demographic characteristics of informants.

\begin{tabular}{|c|c|c|c|c|c|c|c|c|c|c|c|c|c|}
\hline \multirow{2}{*}{$\begin{array}{l}\text { Demographical } \\
\text { characteristics }\end{array}$} & \multicolumn{2}{|c|}{ Sex } & \multicolumn{5}{|c|}{ Age (in years) } & \multicolumn{4}{|c|}{ Education level } & \multicolumn{2}{|c|}{ dwellers } \\
\hline & w & $M$ & $<30$ & $30-40$ & $40-50$ & $50-60$ & $>60$ & II & $\operatorname{Pr}$ & Se & Un & V & $\mathrm{C}$ \\
\hline Number of informants & 136 & 126 & 28 & 62 & 52 & 83 & 37 & 104 & 33 & 63 & 62 & 198 & 64 \\
\hline Percentage & 50 & 48 & 10.7 & 23.7 & 19.8 & 31.7 & 14.1 & 39.7 & 12.6 & 24.0 & 23.7 & 75.6 & 24.4 \\
\hline
\end{tabular}

W: Women; M: Men; II: Illiterate level; Pr: Primary level; Sc: Secondary level; Un: university level; V: Villages; C: City. 
Through this survey, it was shown that the exploitation of medicinal plants was highly widespread throughout the study area at a percentage of $89 \%$ of the total population. The knowledge of medicinal plants, their properties, preparations and use were generally acquired following a long experience accumulated and transmitted from generation to generation.

Practitioners of traditional medicine were classified into five unequal age intervals; the highest frequency of use (31.7\%) was shown by the age group 50-60 years old, followed by the age groups 30-40 and 40-50 years old with $23.7 \%$ and $19.8 \%$ respectively, finally, the lowest frequency of use $(10.7 \%)$ was related to the youngest age group below 30 years old.

These percentages are a good illustration of the local traditional medicine knowledge, mainly related to the older population. Indeed, the elderly people are more likely to provide reliable information, because this age category holds much of the ancestral knowledge, orally transmitted. Unfortunately, this knowledge is currently less and less transmitted and tends to disappear as the youngest generation is no more interested in this knowledge due to a completely new lifestyle, the modernization of the quality of life and the development of the pharmaceutical industry. ${ }^{6,4,38-40}$

It was also noticed that both men and women were involved in the traditional medicine, however; women were more informed about medicinal species compared to men ( $52 \%$ versus $48 \%$ ). These findings were concordant with numerous national ethnobotanical studies, reporting women knowledge to be higher than men's herbal medicine $(52-62 \%),{ }^{4,5,25,27}$ also in term of medicinal plants collection, storage and recipes preparation, women were more involved than men. This predominance of women over men can be explained by their responsibilities as mothers, ${ }^{41,42}$ they are the ones who give first aid to their children in cases of illness and know the secrets of the kitchen and the tricks of the healthy meals and domestic chores. ${ }^{17}$

Most of the traditional medicine users in this inquiry were illiterate with a percentage of $39.7 \%$. Fortunately, despite the inverse relationship between the educational level and customs and traditions, the results showed a significant percentage of users with high educational level, namely secondary or university level with $24 \%$ and $23.7 \%$ respectively. Indeed, according to Boughrara and Legseir ${ }^{27}$ these findings are related to the development of herbal medicine culture, the easy exchange of information and the awareness about the benefits of medicinal plants.

Finally, the highest percentage of use was shown by the villagers (75.6\%) against only $24.4 \%$ of city dwellers (Table 1 ).

\section{Medicinal species used}

\section{Most represented botanical family}

The species inventoried in this inquiry belong to 38 families not homogeneously distributed. The Lamiaceae, Asteraceae and Apiaceae were the most reported families with $14.29 \%$ and $12.86 \%$ and $5.71 \%$ respectively (Figure 2).

These results were concordant with several ethnobotanical studies conducted in Algeria, indeed according to Boudjelal et al. ${ }^{6}$ Benarba et al. ${ }^{23}$ Ouelbani et al. ${ }^{28}$ Bouasla and Bouasla ${ }^{4}$ and Miara et al. ${ }^{5}$ Lamiaceae, Asteraceae and Apiaceae were the most commonly used families throughout the country.

According to Güzel et al. ${ }^{8}$ the popularity of Lamiaceae and Asteraceae may be due to their aromatic characteristics and to their wide geographical spread. Several studies have confirmed the benefits of these families, according to Miliauskas et al. ${ }^{43}$ Khled Khoudja et al ${ }^{44}$ Maulidiani et al..$^{45}$ their importance lies in their high phenolic and flavonoid contents, compounds responsible for the antioxidant activity.

In comparison to Lamiaceae and Asteraceae, the remaining families such as Asphodelaceae, Cupressaceae, Ericaceae, Fabaceae, Fagaceae, Myrtaceae, Oleaceae, Rhamnaceae accounted for $2.86 \%$ each, while Amaryllidaceae, Anacardiaceae, Aristolochiaceae, Asparagaceae Boraginaceae, Brassicaceae, Cistaceae, Cucurbitaceae, Cytinaceae, Gentianaceae, Iridaceae, Lauraceae, Linaceae, Malvaceae, Nitrariaceae, Papaveraceae, Pinaceae, Plantaginaceae and Urticaceae accounted for only $1.43 \%$ each.

Finally, in addition to their therapeutic role, the herbaceous families provide a favorable environment for the germination of the shrub layer seeds and the growth of young seedlings. ${ }^{46}$

\section{Most frequently used species}

Among the 38 families, 70 species were described as medicinal plant in the study area the most cited and used were Tetraclinis articulata, Pistacia lentiscus, Myrtus communis and Thymus vulgaris with $6.49 \%$, $6.11 \%, 5.34 \%$ and $4.96 \%$ respectively (Figure 3 ).

Although the Lamiaceae and Asteraceae families were the most commonly used in the region, only few of their species were among the most cited; indeed, the results showed that the part of Lamiaceae and Asteraceae species did not exceed a percentage of use of $3 \%$.

Regarding Tetraclinis articulata (6.49\%), called Aaraar by the local population, the leaves were mostly used in the form of infusion or

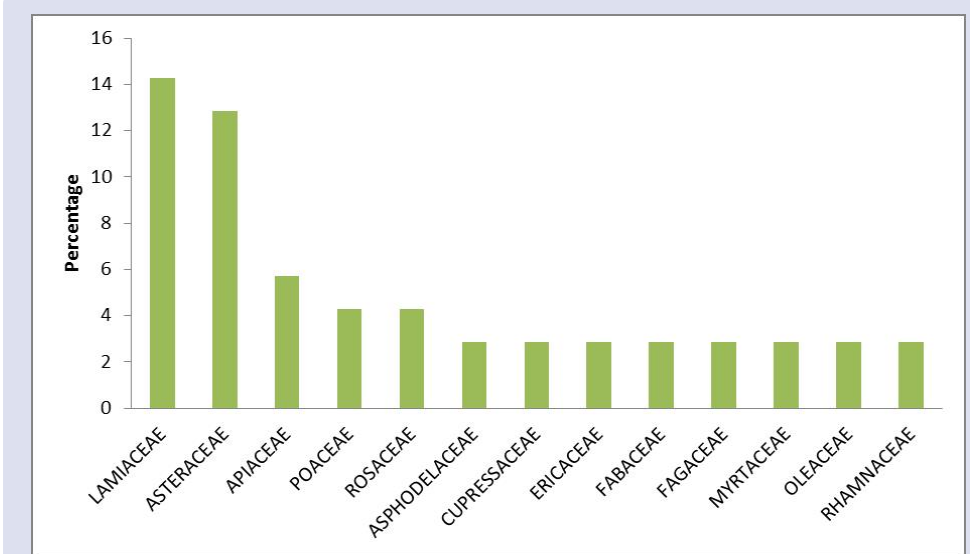

Figure 2: Most represented botanical families. 
decoction to treat several digestive problems and metabolic disorders including diabetes. In addition to these properties the leaves were also confirmed to be effective against Hypoglycemia and hypotension while the seeds were efficient against Hypoglycemia, ${ }^{20,21}$ In comparison to Algeria, Tetraclinis articulata is used in the same ways and treatments in Morocco (anti-diabetic and anti-hypertensive). ${ }^{17}$

According to Gonzalez-Tejero et al. ${ }^{47}$ this species is recommended against respiratory system diseases, moreover, according to Ziyyat et al. ${ }^{48} \mathrm{El}$ Moussaouiti et al. ${ }^{49}$ Bourkhiss et al..$^{50}$ Talbaoui et al.${ }^{51}$ besides its anti-hypertensive and anti-diabetic virtues, this plant is characterized by a high antibacterial activity.

Pistacia lentiscus $(6.11 \%)$ was mainly used against the digestive and the respiratory system diseases. According to several studies this species is characterized by a high antioxidant capacity, the leaves are an important source of flavonoids, tannins, phenolic compounds and a very good source of natural pigments, ${ }^{52-53}$ the extracts isolated from the aerial parts of this species have shown the presence of Terpineol, a substance known by its significant antimicrobial activity ${ }^{54}$ and its ability to inhibit the Mycelian growth..$^{55}$

When it comes to Myrtus communis, called Rayhan, it was mainly indicated locally in the treatment of digestive system diseases, in this context, the same treatment was reported by Gonzales-Terejo et al. ${ }^{47}$ in Italy. According to Bouasla and Bouasla, ${ }^{4}$ this plant is used in the form of infusion or decoction to treat hypotension, diabetes, rheumatism, diarrhea and anxiety in the Northeastern part of Algeria. In Morocco this species is traditionally used for the treatment of respiratory system diseases ${ }^{47}$ and it is reported to be antihyperglycemic. ${ }^{56}$ In Cyprus, Myrtus communis is known for the treatment of skin problems. Furthermore, the leaves and fruits extracts of this plant have been described as hypoglycemic and anti-inflammatory. ${ }^{21}$

Thymus vulgaris, called Zaater by the local population, was locally used in the form of infusion or decoction to treat genitourinary disorders, respiratory system troubles and certain digestive system diseases. An earlier study conducted by Bouzabata ${ }^{21}$ in Souk Ahras in the Eastern part of Algeria showed that this species was used as stomachic, antiseptic and antispasmodic. Thymus vulgaris was also reported to be traditionally used as anti-diabetic in the South-East of Algeria. ${ }^{26}$ Indeed, according to Hyun et al. ${ }^{57}$ the Methanolic extract and the Ethyle Acetate fraction contained in this species play a strong antidiabetic activity. Furthermore, numerous studies have confirmed that Thymol and Carvacol isolated from this species exhibited anti-inflammatory effects. ${ }^{4,58-61}$

This inquiry also showed that Anthemis arvensis and Mentha pulegium were mostly used to treat respiratory diseases, Lavandula stoechas was frequently used for the treatment of digestive system diseases, while Quercus ilex was used in the case of digestive system diseases and rheumatic diseases. Finally, when used in the form of decoction, Tetraclinis articulata was a very effective remedy against flu, coughs and even rheumatism according to the local population. Other species mentioned by the local population according to their ethnopharmacological uses are presented in Table 2.

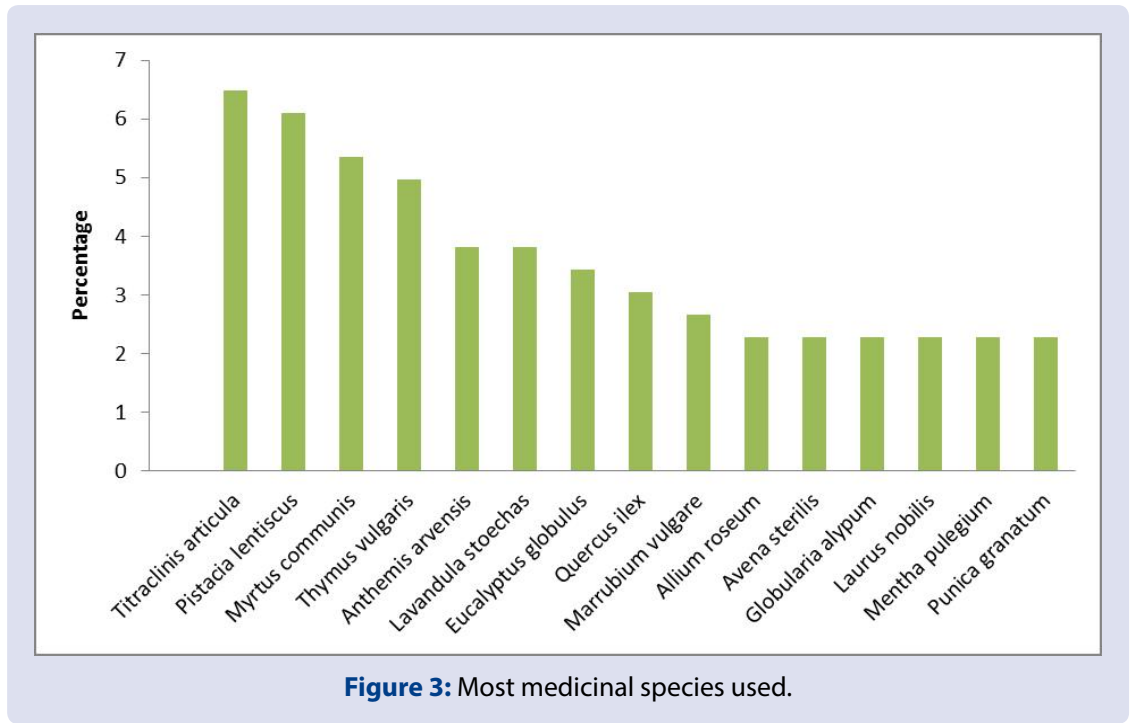

Table 2: List of medicinal plants and their related information.

\begin{tabular}{|c|c|c|c|c|c|c|}
\hline Scientific name and FAMILY & Local names & Part used & $\begin{array}{l}\text { Method of } \\
\text { preparation }\end{array}$ & $\begin{array}{l}\text { Mode of } \\
\text { administ } \\
\text { ration }\end{array}$ & Uses & $\begin{array}{c}\text { Previous } \\
\text { ethnobotanical } \\
\text { literature records } \\
\text { in Algeria } \\
\end{array}$ \\
\hline $\begin{array}{l}\text { Ajuga iva (L.) Schreb. } \\
\text { LAMIACEAE }\end{array}$ & Chendgoura & $\begin{array}{l}\text { Le. } \\
\text { A.pa. }\end{array}$ & $\begin{array}{l}\text { Dec., } \\
\text { Inf. }\end{array}$ & Ora. & $\begin{array}{c}\text { colon ailments, } \\
\text { stomachache, diabetes }\end{array}$ & $\begin{array}{c}6,21,23,26,28 \\
47,62-63\end{array}$ \\
\hline $\begin{array}{l}\text { Allium roseum } \mathrm{L} \text {. } \\
\text { AMARYLLIDACEAE }\end{array}$ & Elthoum & $\begin{array}{l}\text { Bu., } \\
\text { A.pa. }\end{array}$ & $\begin{array}{l}\text { Ckd.Inf. } \\
\text { Pou.,Oth. }\end{array}$ & $\begin{array}{l}\text { Ora.,Mas. } \\
\text { Rin. }\end{array}$ & $\begin{array}{l}\text { hypertension, } \\
\text { stomachache,intoxications,cold, } \\
\text { Cough, influenza,dental square }\end{array}$ & 4,5 \\
\hline $\begin{array}{l}\text { Aloe vera (L.) Burm.f. } \\
\text { ASPHODELACEAE }\end{array}$ & Elsabar & Fl. & Oth. & Oth. & acne, hair loss & 26 \\
\hline $\begin{array}{l}\text { Ammoides pusilla (Brot.) } \\
\text { Breistr. APIACEAE }\end{array}$ & Elnankha & Se. & Oth. & Ora. & menstrual cramps & \\
\hline $\begin{array}{l}\text { Ampelodesmos mauritanicus } \\
\text { (Poir.) T.Durand \& Schinz } \\
\text { POACEAE }\end{array}$ & Eldiss & St. & Dec. & Rin. & Rhumatism, joints inflammation & 6,63 \\
\hline
\end{tabular}




\begin{tabular}{|c|c|c|c|c|c|c|}
\hline Scientific name and FAMILY & Local names & Part used & $\begin{array}{l}\text { Method of } \\
\text { preparation }\end{array}$ & $\begin{array}{l}\text { Mode of } \\
\text { administ } \\
\text { ration }\end{array}$ & Uses & $\begin{array}{c}\text { Previous } \\
\text { ethnobotanical } \\
\text { literature records } \\
\text { in Algeria } \\
\end{array}$ \\
\hline $\begin{array}{l}\text { Anthemis arvensis } \mathrm{L} . \\
\text { ASTERACEAE }\end{array}$ & Elbabounej & $\begin{array}{l}\text { Fl.,Le. } \\
\text { A.pa. }\end{array}$ & $\begin{array}{l}\text { Inf.,Dec. } \\
\text { Pou.,Oth. }\end{array}$ & $\begin{array}{l}\text { Ora.,Oth. } \\
\text { Rin. }\end{array}$ & $\begin{array}{l}\text { Migraine,nose allergy, sneeze,Hair } \\
\text { loss,anxiety, } \\
\text { Insomnia,joints pains,pulmonary allergy, } \\
\text { sore throat (pharyngitis) }\end{array}$ & 6,5 \\
\hline $\begin{array}{c}\text { Anvillea radiata Coss. \& } \\
\text { Durieu } \\
\text { ASTERACEAE }\end{array}$ & Elnagd & Fl. & Dec. & Ora. & Diabetes & 63,26 \\
\hline $\begin{array}{l}\text { Arbutus unedo L. } \\
\text { ERICACEAE }\end{array}$ & Lanj & Fr. & Oth. & Ora. & hypotension & $27,47,63$ \\
\hline $\begin{array}{c}\text { Arisarum vulgare O.Targ.Tozz. } \\
\text { ARACEAE }\end{array}$ & Elbgouga & Se. & Inf. & Ora. & constipation & \\
\hline $\begin{array}{l}\text { Aristolochia baetica L. } \\
\text { ARISTOLOCHIACEAE }\end{array}$ & Borestom & A.pa.,Ro. & Oth. & Mas.,Ora. & wound,Tumors, cancers & 6,63 \\
\hline $\begin{array}{l}\text { Asparagus acutifolius } \mathrm{L} \text {. } \\
\text { ASPARAGACEAE }\end{array}$ & Elzbiradj & St. & Dec. & Rin. & skin allergy,Migraine & $23-24,47,62$ \\
\hline $\begin{array}{l}\text { Asphodelus ramosus } \mathrm{L} . \\
\text { ASPHODELACEAE }\end{array}$ & Elbarwag & Bu.,Ro. & Pou.,Oth. & Oth. & haemorrhoids,acne & $4,27,47$ \\
\hline $\begin{array}{l}\text { Atriplex halimus L. } \\
\text { AMARANTHACEAE }\end{array}$ & Elktaff & Le. & Dec. & Ora. & intoxications & $5-6,26,28$ \\
\hline $\begin{array}{l}\text { Avena sterilis } \mathrm{L} . \\
\text { POACEAE }\end{array}$ & Elchoufan & Se. & Oth. & Ora. & $\begin{array}{l}\text { Colon ailments,hypercholesterolemia, } \\
\text { Diabetes, hypertension, obesity }\end{array}$ & \\
\hline $\begin{array}{l}\text { Borago officinalis } \mathrm{L} \text {. } \\
\text { BORAGINACEAE }\end{array}$ & Lsan elthour & Ro., Le. & $\begin{array}{l}\text { Inf.,Dec. } \\
\text { Pou. }\end{array}$ & Ora., Mas. & $\begin{array}{l}\text { Depression,memory and neurological } \\
\text { disorders, } \\
\text { joints inflammation,gynecological problems }\end{array}$ & $23,27,47$ \\
\hline $\begin{array}{l}\text { Bunium bulbocastanum } \mathrm{L} . \\
\text { APIACEAE }\end{array}$ & Talghouda & A.pa.,Ro. & Oth. & Ora. & $\begin{array}{l}\text { Asthma,pulmonary allergy, } \\
\text { sore throat (pharyngitis),bronchitis }\end{array}$ & 5,23 \\
\hline $\begin{array}{l}\text { Calendula arvensis } \mathrm{L} . \\
\text { ASTERACEAE }\end{array}$ & Eldjomira & Le.,Ro. & Dec., Inf. & Ora. & Rhumatism & \\
\hline $\begin{array}{l}\text { Capparis spinosa } \mathrm{L} . \\
\text { CAPPARACEAE }\end{array}$ & Lakabbar & Ro. & Pou. & Mas. & joints pains,eczema & $26,28,63$ \\
\hline $\begin{array}{l}\text { Carlina gummifera }(\text { L.) Less. } \\
\text { ASTERACEAE }\end{array}$ & Leddad & Le.,Ba.,Ro. & Oth.,Pou. & Oth. & Spirituel,prostate infection & 6 \\
\hline $\begin{array}{l}\text { Centaurium erythraea Rafn } \\
\text { GENTIANACEAE }\end{array}$ & $\begin{array}{l}\text { Merradh el } \\
\text { hnach }\end{array}$ & Le.,A.pa. & Inf.,Pou. & Ora.,Oth. & Diabetes & 6,28 \\
\hline $\begin{array}{l}\text { Ceratonia siliqua } \mathrm{L} \text {. } \\
\text { FABACEAE }\end{array}$ & Elkharoub & Fr. & Oth. & Ora. & stomachache,diarrhoea & 4,28 \\
\hline $\begin{array}{l}\text { Cistus monspeliensis } \mathrm{L} \text {. } \\
\text { CISTACEAE }\end{array}$ & Touzala & A.pa.,Le. & Dec.,Oth. & Ora.,Oth. & $\begin{array}{l}\text { Diabetes,colon ailments,stomachache, } \\
\text { kidney inflammations, wound }\end{array}$ & \\
\hline $\begin{array}{l}\text { Citrullus colocynthis (L.) } \\
\text { Schrad. CUCURBITACEAE }\end{array}$ & Handhal & A.pa. & Oth. & Mas. & haemorrhoids & $5-6,26,28,47,63$ \\
\hline $\begin{array}{c}\text { Clinopodium nepeta }(\mathrm{L} .) \\
\text { Kuntze } \\
\text { LAMIACEAE }\end{array}$ & Elnabta & A.pa.,St. & Dec.,Inf. & Ora. & $\begin{array}{l}\text { Colon ailments,stomachache,influenza, } \\
\text { heart problems }\end{array}$ & \\
\hline $\begin{array}{l}\text { Crataegus oxyacantha } \mathrm{L} \text {. } \\
\text { ROSACEAE }\end{array}$ & Bab adjina & Le. & Dec. & Ora. & anxiety & $4,27-28,47,63$ \\
\hline $\begin{array}{l}\text { Cupressus sempervirens } \mathrm{L} \text {. } \\
\text { CUPRESSACEAE }\end{array}$ & Elbostan & Le. & Dec. & Ora. & haemorrhoids & 28,47 \\
\hline $\begin{array}{l}\text { Cytinus hypocistis }(\mathrm{L} .) \mathrm{L} . \\
\text { CYTINACEAE }\end{array}$ & Daghmous & Fl. & Oth. & Oth. & Heart problems & \\
\hline $\begin{array}{l}\text { Cytisus villosus Pourr. } \\
\text { FABACEAE }\end{array}$ & $\begin{array}{l}\text { Khiyatat lejrah, } \\
\text { ratba }\end{array}$ & A.pa., Le. & $\begin{array}{l}\text { Pou., Inf. } \\
\text { Oth. }\end{array}$ & Oth., Ora. & $\begin{array}{l}\text { wound, stomach ulcer, colon ailments, } \\
\text { stomachache }\end{array}$ & 27 \\
\hline $\begin{array}{l}\text { Dittrichia viscosa }(\mathrm{L} .) \text { Greuter } \\
\text { ASTERACEAE }\end{array}$ & Magraman & $\begin{array}{l}\text { A.pa. } \\
\text { Le. }\end{array}$ & Pou., Oth. & Mas.,Oth., Rin. & Rhumatism, Seborrhea, joints pains & $4,27-28,47,63$ \\
\hline Erica arborea L. ERICACEAE & Elkhlilanj & St. & Inf., Dec. & Ora. & $\begin{array}{l}\text { renal lithiasis, urinary infections, } \\
\text { kidney inflammations, Pinworm infection, } \\
\text { stomachache }\end{array}$ & 23 \\
\hline $\begin{array}{l}\text { Eucalyptus globulus Labill. } \\
\text { MYRTACEAE }\end{array}$ & Elkalytous & Le. & Inf., Dec. & Ora., Oth. & $\begin{array}{c}\text { Cold, Cough,bronchitis, } \\
\text { pulmonary allergy,Rhumatism, } \\
\text { nose allergy, sneeze,renal lithiasis }\end{array}$ & $4,6,26-28,47$ \\
\hline $\begin{array}{l}\text { Ferula communis } \mathrm{L} . \\
\text { APIACEAE }\end{array}$ & Elawsaj & Le. & Oth. & Oth. & skin allergy & 6 \\
\hline $\begin{array}{l}\text { Gladiolus byzantinus Mill. } \\
\text { IRIDACEAE }\end{array}$ & Baslate etdib & $\mathrm{Bu}$. & Dec. & Rin. & female infertility & \\
\hline
\end{tabular}




\begin{tabular}{|c|c|c|c|c|c|c|}
\hline Scientific name and FAMILY & Local names & Part used & $\begin{array}{l}\text { Method of } \\
\text { preparation }\end{array}$ & $\begin{array}{l}\text { Mode of } \\
\text { administ } \\
\text { ration }\end{array}$ & Uses & $\begin{array}{l}\text { Previous } \\
\text { ethnobotanical } \\
\text { literature records } \\
\text { in Algeria } \\
\end{array}$ \\
\hline $\begin{array}{l}\text { Globularia alypum L. } \\
\text { PLANTAGINACEAE }\end{array}$ & Tassalgha & Le. & Dec. & Ora. & Diabetes,influensa, gases, jaundice & $4,6,26,28,47,63-64$ \\
\hline $\begin{array}{l}\text { Hordeum murinum } \mathrm{L} . \\
\text { POACEAE }\end{array}$ & Sboulet el far & Le. & Inf. & Ora. & jaundice & \\
\hline $\begin{array}{l}\text { Laurus nobilis L. } \\
\text { LAURACEAE }\end{array}$ & Elrand & Le. & $\begin{array}{l}\text { Dec., Inf. } \\
\text { Oth. }\end{array}$ & Ora., Mas. & hypertension, hair loss & $4,27-28,47,63$ \\
\hline $\begin{array}{l}\text { Lavandula stoechas } \mathrm{L} \text {. } \\
\text { LAMIACEAE }\end{array}$ & $\begin{array}{l}\text { Halhal, } \\
\text { elkhozama }\end{array}$ & $\begin{array}{l}\text { Fl., St. } \\
\text { Le. }\end{array}$ & $\begin{array}{l}\text { Dec., Inf. } \\
\text { Oth., Ckd. }\end{array}$ & Mas.,Ora., Oth. & $\begin{array}{l}\text { gases, anxiety,Heart problems, } \\
\text { stomachache,Rhumatism, } \\
\text { gynecological problems,pulmonary allergy }\end{array}$ & $4,27-28,47$ \\
\hline $\begin{array}{l}\text { Linum usitatissimum } \mathrm{L} \text {. } \\
\text { LINACEAE }\end{array}$ & Elkettan & Se. & Dec., Oth. & Ora., Rin., Oth. & $\begin{array}{l}\text { emaciation, constipation, } \\
\text { hypercholesterolemia,hypertension, } \\
\text { pulmonary allergy,colon } \\
\text { ailments,stomachache, } \\
\text { earache,hair loss }\end{array}$ & 4,28 \\
\hline $\begin{array}{l}\text { Lonicera implexa Aiton } \\
\text { CAPRIFOLIACEAE }\end{array}$ & Chahm elatrous & Le. & Oth., Dec. & Oth., Ora. & skin allergy eyes,enuresis & \\
\hline $\begin{array}{l}\text { Malva sylvestris L. } \\
\text { MALVACEAE }\end{array}$ & Elkhobiz & Le.,Ro. & $\begin{array}{l}\text { Ckd.,Inf. } \\
\text { Dec. }\end{array}$ & Ora., whi. & $\begin{array}{l}\text { hypertension, stomachache, Tooth } \\
\text { ache, } \\
\text { urinary infections }\end{array}$ & $4,6,28,47$ \\
\hline $\begin{array}{l}\text { Marrubium vulgare L. } \\
\text { LAMIACEAE }\end{array}$ & Timeriwat & $\begin{array}{l}\text { Le., Fl., } \\
\text { A.pa., St. }\end{array}$ & $\begin{array}{l}\text { Dec., Inf. } \\
\text { Ckd. }\end{array}$ & Oth., Ora. & $\begin{array}{l}\text { Infertility, probleme des dents, Colon } \\
\text { ailments, } \\
\text { enuresis,haemorrhoids,Tumors, } \\
\text { cancers,rhumatism }\end{array}$ & $6,26,28,47,63$ \\
\hline $\begin{array}{l}\text { Mentha pulegium L. } \\
\text { LAMIACEAE }\end{array}$ & Elfliou & Le. & $\begin{array}{l}\text { Dec., Inf. } \\
\text { Pou. }\end{array}$ & Ora., Oth. & $\begin{array}{l}\text { Asthma, Migraine,anxiety,colon ailments, } \\
\text { stomachache }\end{array}$ & $4,6,28,47$ \\
\hline $\begin{array}{l}\text { Crataegus germanica }(\mathrm{L} .) \\
\text { Kuntze ROSACEAE }\end{array}$ & Elzaarour & Fl.,Le. & Dec. & Ora. & Heart problems,stomachache & 4 \\
\hline $\begin{array}{l}\text { Myrtus communis L. } \\
\text { MYRTACEAE }\end{array}$ & Elrayhan & Le. & $\begin{array}{l}\text { Dec., Inf. } \\
\text { Pou. }\end{array}$ & Ora., Oth. & $\begin{array}{l}\text { anxiety, gases, stomach ulcer, } \\
\text { Throat of the nose,colon ailments, } \\
\text { stomachache,constipation, anxiety, } \\
\text { epistaxis,Asthma,Tooth ache }\end{array}$ & $\begin{array}{c}4,6,23,27-28,47,63 \\
65\end{array}$ \\
\hline $\begin{array}{l}\text { Nepeta multibracteata Desf. } \\
\text { LAMIACEAE }\end{array}$ & Timrsad & Le., A.pa. & Oth. & Ora., Oth. & gynecological problems, joints pains & \\
\hline Olea europaea L. OLEACEAE & Elzebouj & Le. & Dec., Inf. & Ora., whi. & Rhumatism, gingivitis, tooth abscess & $4,6,26-28,47,63$ \\
\hline $\begin{array}{l}\text { Opuntia ficus-indica (L.) Mill. } \\
\text { CACTACEAE }\end{array}$ & $\begin{array}{l}\text { Karmous nsara, } \\
\text { hendi }\end{array}$ & St. & Oth. & Mas. & eczema & 27,47 \\
\hline $\begin{array}{l}\text { Papaver rhoeas } \mathrm{L} . \\
\text { PAPAVERACEAE }\end{array}$ & Benaaman & A.pa. & Dec. & Ora. & memory and neurological disorders & 28,47 \\
\hline $\begin{array}{l}\text { Peganum harmala L. } \\
\text { NITRARIACEAE }\end{array}$ & Elharmel & Se. & Dec., Oth. & Ora. & stomachache, joints pains & $4-6,26,28,47,64$ \\
\hline $\begin{array}{l}\text { Phillyrea latifolia } \mathrm{L} . \\
\text { OLEACEAE }\end{array}$ & Elktem & Le. & Inf.,Pou. & Ora., Oth. & stomachache, hair color & \\
\hline $\begin{array}{l}\text { Pinus halepensis Mill. } \\
\text { PINACEAE }\end{array}$ & Elsnoubar & Se. & Oth. & Ora. & stomachache,kidney inflammations & $4,23-24,47,63,66$ \\
\hline $\begin{array}{l}\text { Pistacia lentiscus L. } \\
\text { ANACARDIACEAE }\end{array}$ & Eldharou & $\begin{array}{l}\text { Le., Se., } \\
\text { A.pa., }\end{array}$ & $\begin{array}{l}\text { Oth., Dec. } \\
\text { Inf., Pou. } \\
\text { Oth., Ckd. }\end{array}$ & Mas., Ora. & $\begin{array}{l}\text { Cough, Nervous colon, burns, } \\
\text { Acne, skin allergy, wound, stomach ulcer, } \\
\text { vomiting, stomachache, diarrhea,bronchitis, } \\
\text { pulmonary allergy, earache }\end{array}$ & $4-5,27-28,47,63$ \\
\hline $\begin{array}{l}\text { Punica granatum } \mathrm{L} . \\
\text { LYTHRACEAE }\end{array}$ & Kchour elroman & Ba. & $\begin{array}{l}\text { Oth., Dec. } \\
\text { Inf., Pou. } \\
\text { Oth., Ckd. }\end{array}$ & Ora., Oth. & $\begin{array}{l}\text { Colon ailments, wound,stomach ulcer, } \\
\text { diarrhoea,cough }\end{array}$ & $4,26-28,47$ \\
\hline Quercus ilex L. FAGACEAE & Elbalout & $\begin{array}{l}\text { Ba., Le. } \\
\text { Fr. }\end{array}$ & $\begin{array}{l}\text { Dec., Oth. } \\
\text { Ckd. }\end{array}$ & Ora. & $\begin{array}{l}\text { enuresis,Cough,stomach ulcer, } \\
\text { haemorrhoids, } \\
\text { Colon ailments,stomachache } \\
\text { Diabetes,Bladder problems }\end{array}$ & 47,63 \\
\hline Quercus suber L. FAGACEAE & Elfernan & Ba. & Oth. & Oth. & stomachache, enuresis,epistaxis & \\
\hline $\begin{array}{l}\text { Rhamnus alaternus } \mathrm{L} . \\
\text { RHAMNACEAE }\end{array}$ & Mlilles & St., Ba. & Dec. & Ora. & jaundice & $5-6,23-24,28,47,66$ \\
\hline $\begin{array}{l}\text { Rosmarinus officinalis } \mathrm{L} \text {. } \\
\text { LAMIACEAE }\end{array}$ & Iklil eldjabel & Le., A.pa. & Dec., Inf. & Ora., Rin. & $\begin{array}{l}\text { hypertension, Hair loss,eczema, } \\
\text { colon ailments,stomachache }\end{array}$ & $4-6,26-28,47,63$ \\
\hline $\begin{array}{l}\text { Rubus ulmifolius Schott } \\
\text { ROSACEAE }\end{array}$ & Elaolig & St.,Fr. & Dec., Oth. & Ora., Oth. & scabies, Acne & 27,47 \\
\hline
\end{tabular}




\begin{tabular}{|c|c|c|c|c|c|c|}
\hline Scientific name and FAMILY & Local names & Part used & $\begin{array}{l}\text { Method of } \\
\text { preparation }\end{array}$ & $\begin{array}{l}\text { Mode of } \\
\text { administ } \\
\text { ration }\end{array}$ & Uses & $\begin{array}{l}\text { Previous } \\
\text { ethnobotanical } \\
\text { literature records } \\
\text { in Algeria } \\
\end{array}$ \\
\hline $\begin{array}{l}\text { Ruta chalepensis } \mathrm{L} . \\
\text { RUTACEAE }\end{array}$ & Fidjel & A.pa., Le. & Dec. & Ora. & Insomnia, phobia, depression, anxiety & $6,24,23-24,47,63$ \\
\hline $\begin{array}{l}\text { Salvia officinalis L. } \\
\text { LAMIACEAE }\end{array}$ & Sauja & $\begin{array}{l}\text { Se., Le. } \\
\text { A.pa. }\end{array}$ & Inf., Oth. & Ora. & emaciation, stomachache, diarrhoea & $4,6,26,28,47,63$ \\
\hline $\begin{array}{l}\text { Scolymus hispanicus L. } \\
\text { ASTERACEAE }\end{array}$ & Guernina & $\begin{array}{l}\text { A.pa. } \\
\text { St. }\end{array}$ & Dec., Oth. & Ora. & $\begin{array}{c}\text { colon ailments,stomachache, } \\
\text { memory and neurological disorders, } \\
\text { Rhumatism }\end{array}$ & \\
\hline $\begin{array}{l}\text { Silybum marianum (L.) } \\
\text { Gaertn. } \\
\text { ASTERACEAE }\end{array}$ & Elkhanfra & Fl. & Inf. & Ora. & hypotension & 5 \\
\hline $\begin{array}{l}\text { Sinapis arvensis } \mathrm{L} . \\
\text { BRASSICACEAE }\end{array}$ & Elkhardel & Se. & Oth. & Mas. & sciatica & \\
\hline $\begin{array}{l}\text { Sonchus arvensis L. } \\
\text { ASTERACEAE }\end{array}$ & Elaasloudj & St. & Oth. & Ora. & anaemia & \\
\hline $\begin{array}{c}\text { Taraxacum officinale } \\
\text { F.H.Wigg. ASTERACEAE }\end{array}$ & Elhendbaa & Le., Ro. & Inf., Dec. & Ora. & $\begin{array}{l}\text { colon ailments,stomachache, } \\
\text { constipation }\end{array}$ & 6 \\
\hline $\begin{array}{l}\text { Teucrium polium L. } \\
\text { LAMIACEAE }\end{array}$ & Eldjaida & Le. & Inf. & Ora. & stomachache & $4-6,26,28,47,63$ \\
\hline $\begin{array}{l}\text { Thymus vulgaris } \mathrm{L} \text {. } \\
\text { LAMIACEAE }\end{array}$ & Elzaater & A.pa., Le. & Oth., Dec. & Ora., Rin., whi. & $\begin{array}{c}\text { Pinworm infection, influenza } \\
\text {,female infertility ,urinary infections, Cold, } \\
\text { Cough, } \\
\text { Tooth ache, Asthma, joints pains } \\
\text { sore throat (pharyngitis), } \\
\text { gynecological problems, } \\
\text { Bladder problems, } \\
\text { urinary infections, } \\
\text { hypercholesterolemia, }\end{array}$ & 4,26 \\
\hline $\begin{array}{l}\text { Tetraclinis articulata (Vahl) } \\
\text { Mast. } \\
\text { CUPRESSACEAE }\end{array}$ & Aarar & $\begin{array}{l}\text { Le.,A.pa., } \\
\text { Fr. }\end{array}$ & $\begin{array}{l}\text { Dec., Inf. } \\
\text { Pou., Oth. }\end{array}$ & Ora., Oth. & $\begin{array}{l}\text { Anxiety, stomach ulcer, cold } \\
\text { tuberculosis, urinary infections, } \\
\text { vertigo, Migraine, diarrhea, cough, } \\
\text { colon ailments, stomachache }\end{array}$ & \\
\hline $\begin{array}{l}\text { Urtica dioica } \mathrm{L} \text {. } \\
\text { URTICACEAE }\end{array}$ & Horig, el kerras & A.pa. & Dec., Inf. & Ora. & $\begin{array}{l}\text { Rheumatism,anemia, } \\
\text { urinary infections, } \\
\text { kidney inflammations, } \\
\text { renal lithiasis, gases }\end{array}$ & $4,6,27-28,47$ \\
\hline $\begin{array}{l}\text { Ziziphus lotus (L.) Lam. } \\
\text { RHAMNACEAE }\end{array}$ & Sedra & Ro. & Dec. & whi. & Tooth ache & $6,26,28$ \\
\hline
\end{tabular}

Inf: Infusion, Dec: Decoction, Ckd: Cooked, Pou: Poullice, Oth: Others, Ora: Oral, Rin: Rincing, Mas: Massage, Whi: Whitewashing, A.pa: Aerial parts, St: Stem, Le: Leaves, Fl: Flowers, Fr: Fruits, Bu: Bulbs, Ro: Roots, Se: Seeds, Ba: Barks.

\section{Parts used and preparation}

Among all the parts of medicinal plants, leaves were the most-used part throughout the study area with a percentage of $56.95 \%$ (Figure 4 ), in this respect, the predominance of leaves in traditional remedies has been reported by several studies in Algeria, ${ }^{4-5,23,27,28}$ Spain, ${ }^{67}$ Italy $^{68}$ and Turkey. ${ }^{69}$

The preference of leaves in traditional medicine can be explained by the ease and speed of harvest, ${ }^{34,40,70}$ besides, the leaves are the site of photosynthesis and sometimes the storage site of secondary metabolites, responsible for the plant's biological properties. ${ }^{71-73}$

On the other hand, the insignificant use of fruits (2.67\%), bulbs (2.67\%) and roots $(4.58 \%)$ is a very positive behavior, as it avoids the plant digging and guarantees the rapid recovery of the aerial part, similarly, the low use of flowers and fruits allows the natural seed dispersal and thus ensuring the survival of the species.

Decoction was the most frequent form of use in the study area with a percentage of $43.51 \%$ (Figure 5), the same finding was reported by several studies carried out throughout the Mediterranean basin including Algeria.--6,18,40,64,65,74
In the study area, among all the surveyed population, the medicinal plants were orally administered in $71 \%$ of cases (Figure 6), whereas the remaining ways of use such as inhalation and fumigation were used in $18.3 \%$ of cases. In the cases of dermatological and rheumatic diseases, massaging was reported by $5 \%$ of the population.

According to Daoudi et al. ${ }^{75}$ decoction in the form of tea, milk or water is the most common way of use in the cold areas; the decoction time varies from 15 to $20 \mathrm{~min}$ depending on the hardness of the leaves. The same authors report that decoction is the most effective way of use as long as it allows almost the full extraction and the best assimilation of the active compounds in addition to warming the body and disinfecting the plant; however, decoction could destroy some important active compounds.

\section{Therapeutic indication}

This inquiry showed that the majority of medicinal plants were mainly used against gastro-intestinal system diseases with a percentage of $28.24 \%$, Ear, Nose and Throat (ENT) and respiratory diseases (14.12\%), metabolic diseases (11.83\%) and skin conditions (10.69\%) (Figure 7). The same findings have also been highlighted by several 


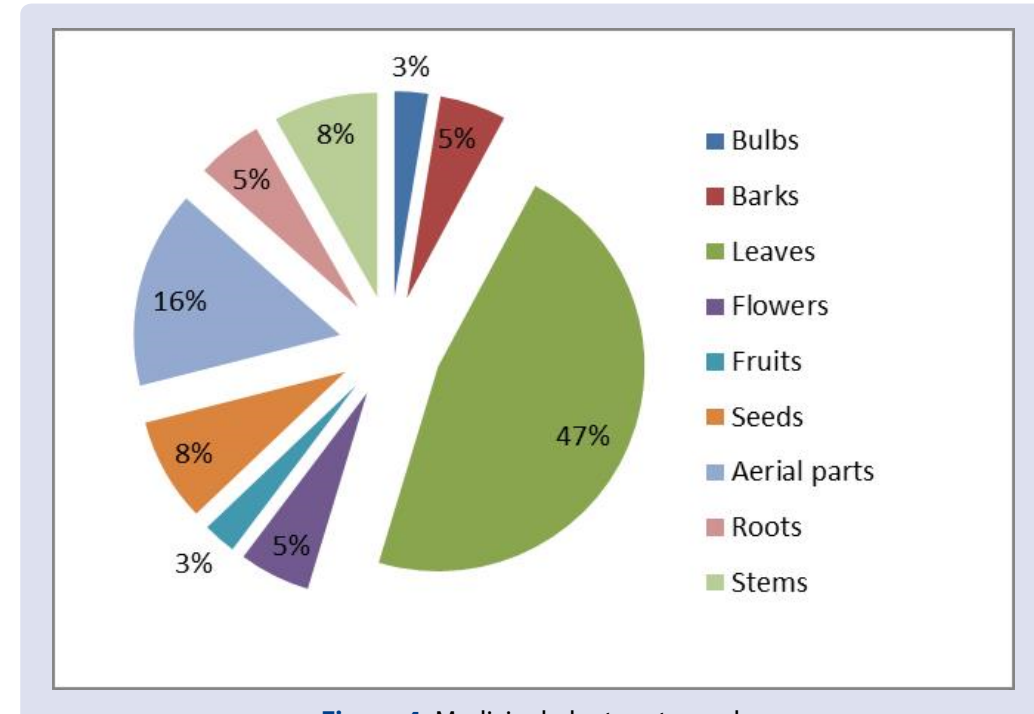

Figure 4: Medicinal plant parts used.

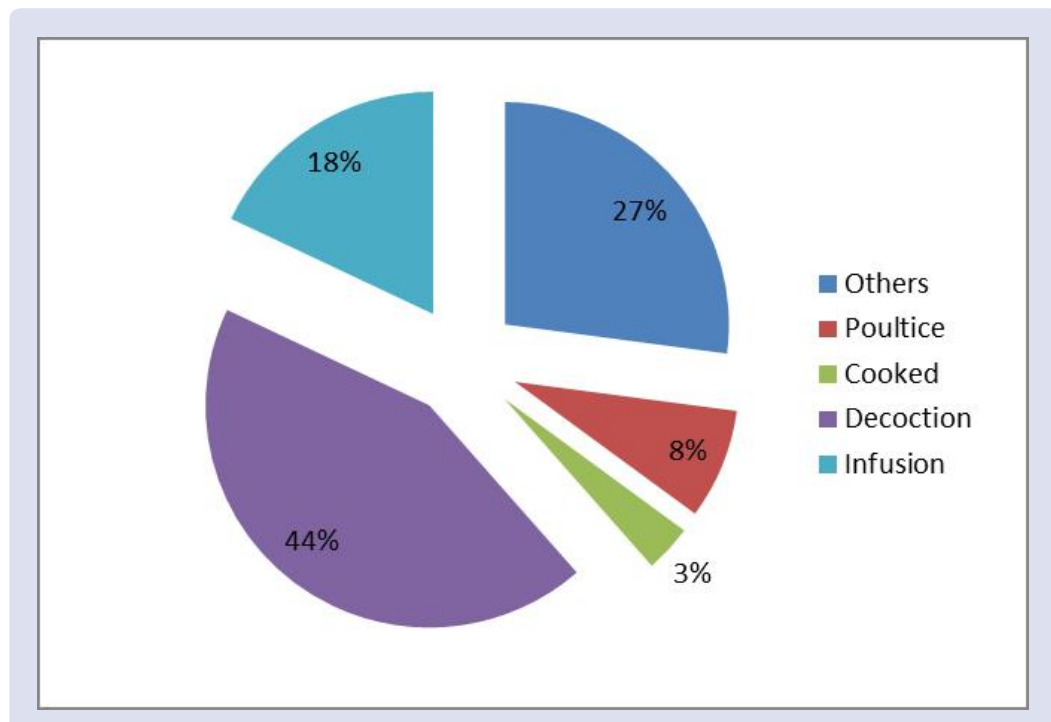

Figure 5: Methods of medicinal plants preparation used.

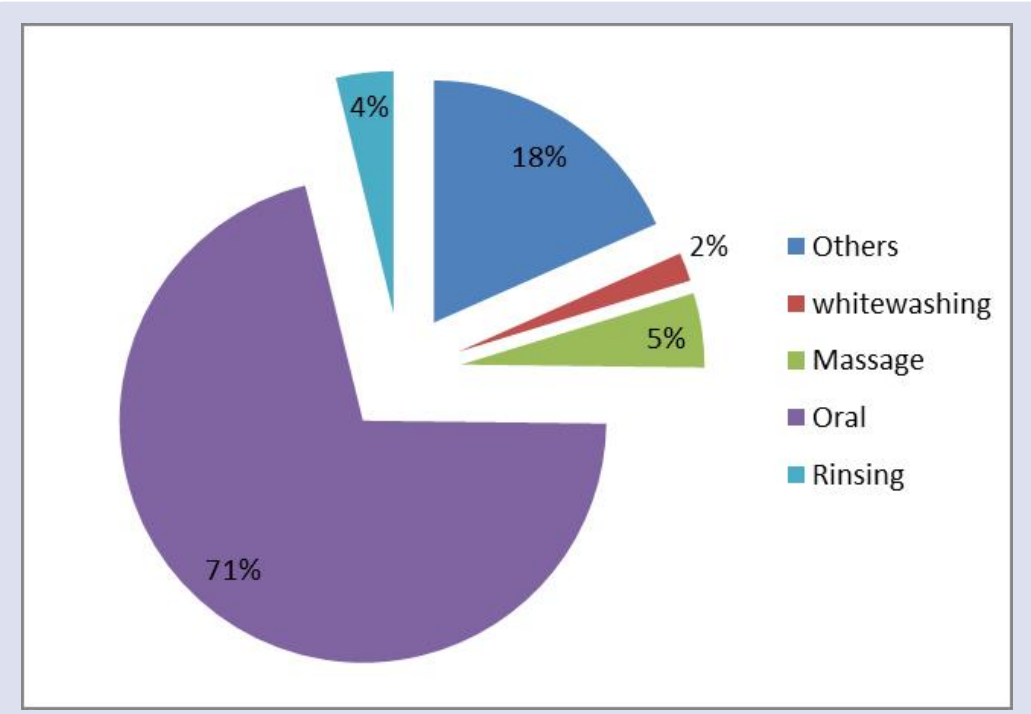

Figure 6: Modes of administration of medicinal plants used. 
studies conducted in Algeria ${ }^{4,5,28,62}$ and all around the Mediterranean basin ${ }^{18,47,56,76,77}$ with slightly different percentages.

\section{Statistical data analysis}

\section{Informant consensus factor (ICF)}

Based on the used reports, the recorded data were classified into 10 different ailment categories (Table 3), the highest ICF values were related to the ENT and respiratory system diseases $(0.67)$ followed by gastro-intestinal system diseases (0.59) and metabolic diseases (0.57), these high ICF values could be explained by the aptitude of informants to identify these categories of diseases known by their very common clinical signs, the simplicity of their treatment using medicinal plants ${ }^{78-83}$ and also by the high incidence of these diseases in the study area. In the same context, osteo-articular diseases, oral cavity diseases and cancer showed the lowest ICF values.

\section{Fidelity level ( $F L)$}

The fidelity level was calculated for the most cited species (15 plants), the higher the FL, the higher the preference of informants to a specific species compared to the rest (Table 4). The highest FL (100\%) was shown for Rhamnus alaternus, a species mainly used in the study area against metabolic diseases, according to Benarba et al. ${ }^{23}$ the same FL of $100 \%$ was shown for this species against hepatic diseases in Mascara in NorthWest Algeria. Indeed, Rhamnus alaternus is considered as hepatic active plant and is used against liver diseases. According to Azaizeh et al. ${ }^{79}$ Ben Ammar et al ${ }^{80}$ Boudjelal et al. ${ }^{6}$ Miara et al.$^{62}$ Khacheba et al ${ }^{81}$ this plant showed an encouraging inhibition of aflatoxin B1-, nifuroxazide-, sodium azide-induced mutagenicity and enzymatic activities of Aspergillus oryzae-amylase involved in hepatic cancers.

In term of skin diseases, the highest FL (25\%) was observed for Pistacia lentiscus. In this context, the same finding was mentioned previously by Bouasla and Bouasla ${ }^{4}$. Regarding ENT and respiratory diseases

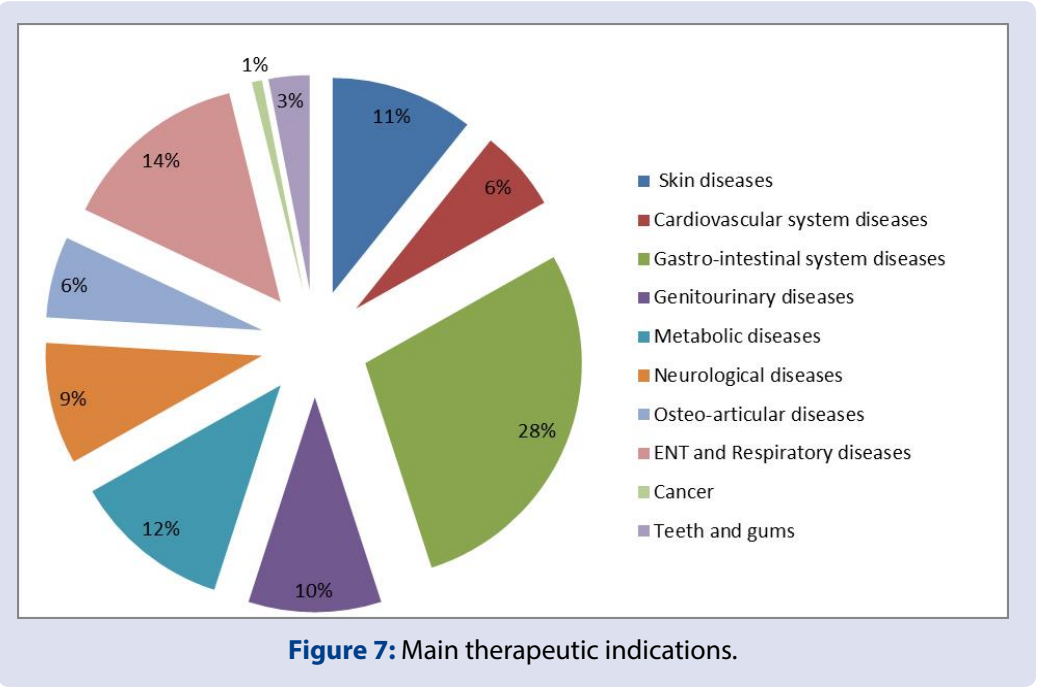

Table 3: Informant Consensus Factor (IFC) per ailment category.

\begin{tabular}{|c|c|c|c|c|}
\hline Category & Ailments/disorders & Number of used reports & Number of Taxa & IFC \\
\hline $\begin{array}{l}\text { Ear, Nose and Throat (ENT) and } \\
\text { respiratory diseases }\end{array}$ & $\begin{array}{l}\text { Cold, cough, asthma, bronchitis, influenza, pulmonary allergy, } \\
\text { tuberculosis, earache, epistaxis, sore throat (pharyngitis), nose } \\
\text { allergy, sneeze }\end{array}$ & 37 & 13 & 0.67 \\
\hline Gastro-intestinal system diseases & $\begin{array}{l}\text { Colon ailments, nervous colon, diarrhoea, gases, constipation, } \\
\text { haemorrhoids, stomach ulcer, stomachache, intoxications, } \\
\text { pinworm infection, vomiting }\end{array}$ & 74 & 31 & 0.59 \\
\hline Metabolic diseases & $\begin{array}{l}\text { Diabetes, hypercholesterolemia, emaciation, obesity, anemia, } \\
\text { jaundice. }\end{array}$ & 31 & 14 & 0.57 \\
\hline Neurological diseases & $\begin{array}{l}\text { Migraine, depression, anxiety, insomnia, vertigo, phobia, } \\
\text { memory and neurological disorders, spiritual }\end{array}$ & 24 & 12 & 0.52 \\
\hline Skin diseases & $\begin{array}{l}\text { wound, burns, hair color, hair loss, acne ,eczema, skin allergy, } \\
\text { scabies, Seborrhea }\end{array}$ & 28 & 19 & 0.33 \\
\hline Cardiovascular system diseases & Heart problems, hypertension, hypotension & 16 & 11 & 0.33 \\
\hline Genitourinary diseases & $\begin{array}{l}\text { Bladder problems, Prostate infection, menstrual cramps, } \\
\text { Infertility, renal lithiasis, urinary infections, enuresis, kidney } \\
\text { inflammations, gynecological problems. }\end{array}$ & 26 & 18 & 0.32 \\
\hline Osteo-articular diseases & Rhumatism, joints inflammation, joints pains, sciatica. & 16 & 13 & 0.20 \\
\hline Teeth and gums & Tooth ache, gingivitis, tooth abscess, dental square & 8 & 7 & 0.14 \\
\hline Cancer & Tumors, cancers & 2 & 2 & 0.00 \\
\hline
\end{tabular}


Table 4: Fidelity level values (FL) for the most medicinal plants used.

\begin{tabular}{|c|c|c|}
\hline Aliment category & Plants & FL (\%) \\
\hline & Pistacia lentiscus & 25.00 \\
\hline \multirow[t]{3}{*}{ Skin diseases } & Anthemis arvensis & 10.00 \\
\hline & Laurus nobilis & 16.67 \\
\hline & Punica granatum & 16.67 \\
\hline \multirow[t]{2}{*}{ Cancer } & Marrubium vulgare & 14.29 \\
\hline & Lavandula stoechas & 10.00 \\
\hline \multirow[t]{3}{*}{ Cardiovascular system diseases } & Marrubium vulgare & 14.29 \\
\hline & Laurus nobilis & 83.33 \\
\hline & Titraclinis articula & 41.18 \\
\hline \multirow[t]{12}{*}{ Gastro-intestinal system diseases } & Pistacia lentiscus & 31.25 \\
\hline & Myrtus communis & 78.57 \\
\hline & Thymus vulgaris & 7.69 \\
\hline & Lavandula stoechas & 40.00 \\
\hline & Quercus ilex & 50.00 \\
\hline & Marrubium vulgare & 28.57 \\
\hline & Avena sterilis & 33.33 \\
\hline & Globularia alypum & 16.67 \\
\hline & Mentha pulegium & 50.00 \\
\hline & Punica granatum & 66.67 \\
\hline & Titraclinis articula & 11.76 \\
\hline & Thymus vulgaris & 38.46 \\
\hline \multirow[t]{6}{*}{ Genitourinary diseases } & Lavandula stoechas & 11.11 \\
\hline & Eucalyptus globulus & 11.11 \\
\hline & Quercus ilex & 37.50 \\
\hline & Marrubium vulgare & 33.33 \\
\hline & Anthemis arvensis & 10.00 \\
\hline & Quercus ilex & 12.50 \\
\hline \multirow[t]{4}{*}{ Metabolic diseases } & Avena sterilis & 66.67 \\
\hline & Globularia alypum & 66.67 \\
\hline & Rhamnus alaternus & 100.00 \\
\hline & Titraclinis articula & 29.41 \\
\hline \multirow[t]{6}{*}{ Neurological diseases } & Myrtus communis & 7.14 \\
\hline & Anthemis arvensis & 30.00 \\
\hline & Lavandula stoechas & 30.00 \\
\hline & Mentha pulegium & 33.33 \\
\hline & Titraclinis articula & 17.65 \\
\hline & Pistacia lentiscus & 43.75 \\
\hline \multirow[t]{7}{*}{ ENT and respiratory diseases } & Myrtus communis & 7.14 \\
\hline & Thymus vulgaris & 46.15 \\
\hline & Anthemis arvensis & 40.00 \\
\hline & Eucalyptus globulus & 88.89 \\
\hline & Globularia alypum & 16.67 \\
\hline & Mentha pulegium & 16.67 \\
\hline & Punica granatum & 16.67 \\
\hline \multirow[t]{3}{*}{ Osteo-articular diseases } & Anthemis arvensis & 10.00 \\
\hline & Lavandula stoechas & 10.00 \\
\hline & Myrtus communis & 7.14 \\
\hline \multirow[t]{2}{*}{ Teeth and gums } & Thymus vulgaris & 7.69 \\
\hline & Marrubium vulgare & 14.29 \\
\hline
\end{tabular}

the highest FL (88.89\%) was shown for Eucalyptus, in this category of diseases the same finding was reported for Eucalyptus globulus by Gonzales-Terejo et al. ${ }^{47}$ in Albania, Benarba et al. ${ }^{23}$ in Mascara ( $\mathrm{FL}=$ $100 \%)$, Ouelbani et al..$^{28}$ in Constantine and Mila ( $\left.\mathrm{FL}=93.93 \%\right)$.

Laurus nobilis was the main species used by the local population against cardiovascular system diseases with a fidelity level of $83.33 \%$. According to Ouelbani et al. ${ }^{28}$ Laurus nobilis recorded in the east side of Algeria an FL of 54.54\% for the treatment of cardiovascular diseases.

Finally, two species received the highest FL in the treatment of gastrointestinal system diseases by the local population, namely Myrtus communis $(\mathrm{FL}=78.57 \%)$ and Punica granatum $(\mathrm{FL}=66.67 \%)$. In this context Ouelbani et al. ${ }^{28}$ and Benarba et al. ${ }^{23}$ reported the same high FL for the two species against gastro-intestinal diseases in Constantine and Mila (East Algeria) and Mascara (West of Algeria). 


\section{CONCLUSION}

This first ethnobotanical study conducted in the region of Bissa revealed a very rich local knowledge in term of traditional herbal medicine; this fact was reflected by the high diversity of species used in the treatment of several diseases. The gastro-intestinal disorders, respiratory troubles, metabolic diseases and skin affections were respectively the most frequently treated ailments and Lamiaceae and Asteraceae were the most commonly used botanical families. In light of this, it is therefore very important to subject some of the major species to further phytochemical and pharmacological studies in order to validate their traditional use and to probably discover new bioactive molecules.

It was also possible through this ethnobotanical study to notice the possibility of using these medicinal plants in local socio-economic development while preserving the floristic richness of the region of Bissa, in the framework of valorization of the human and natural potential.

\section{ACKNOWLEDGMENTS}

We would like to express our most sincere gratitude to the local people of the region of Bissa for their vital role in the successful completion of this survey by sharing their traditional medicine and indigenous knowledge.

\section{CONFLICTS OF INTEREST}

The authors declare no conflicts of interest.

\section{ABBREVIATIONS}

IFC: Informant Consensus Factor; FL: Fidelity Level; ENT: Ear, Nose and Throat; Inf: Infusion, Dec: Decoction; Ckd: Cooked; Pou: Poullice; Oth: Others; Ora: Oral; Rin: Rincing; Mas: Massage; Whi: Whitewashing; A.pa: Aerial parts; St: Stem; Le: Leaves; Fl: Flowers; Fr: Fruits; Bu: Bulbs; Ro: Roots; Se: Seeds; Ba: Barks.

\section{REFERENCES}

1. Balick MJ, Cox PA. Plants, people and culture: The science of ethnobotany. NewYork: Scientific American Library; 1996;228.

2. Prance GT. Ethnobotany and the future of conservation. Biologist (London). 200047(2):65-8.

3. Baba Aissa F. Medicinal plants in Algeria. Identification, description of active ingredient properties and traditional use of common plants in Algeria. Algiers: Bouchene and Adiwan; 1991;180.

4. Bouasla A, Bouasla I. Ethnobotanical survey of medicinal plants in northeastern of Algeria. Phytomedicine. 2017;36:68-81.

5. Miara MD, Bendif H, Ait Hammou M, Teixidor-Toneu I. Ethnobotanical survey of medicinal plants used by nomadic peoples in the Algerian steppe. Journal of Ethnopharmacology. 2018;219:248-56

6. Boudjelal A, Henchiri C, Sari M, Sarri D, Hendel N, Benkhaled A, Ruberto G. Herbalists and wild medicinal plants in M'Sila (North Algeria): An ethnopharmacology survey. Journal of Ethnopharmacology. 2013;148:395-402

7. Kaval I, Behçet L, Cakilcioglu U. Ethnobotanical study on medicinal plants in Geçitli and its surrounding (Hakkari-Turkey). Journal of Ethnopharmacology. 2014;155(1):171-84

8. Güzel Y, Güzelșemme M, Miski M. Ethnobotany of medicinal plants used in Antakya: A multicultural district in Hatay Province of Turkey. Journal of Ethnopharmacology. 2015;174:118-52.

9. Mükemre M, Behçet L, Çakılcıoğlu U. Ethnobotanical study on medicinal plants in villages of Çatak (Van-Turkey). Journal of Ethnopharmacology. 2015;166:36174.

10. Altundağ Çakır E. Traditional knowledge of wild edible plants of Iğdır Province (East Anatolia, Turkey). Acta Soc Bot Pol. 2017;86(4):3568.

11. Chaves DSA, Siqueira RCS, Souza LM, Mirza NG, Sanches MNG, et al. Traditional uses of medicinal plants at Seropédica, Rio de Janeiro. Journal of Pharmacy and Pharmacology Research. 2017;5(1):1-14.
12. Silva HCH, Caraciolo RLF, Marangon LC, Ramos MA, Santos LL, Albuquerque UP. Evaluating different methods used in ethnobotanical and ecological studies to record plant biodiversity. Journal of Ethnobiology and Ethnomedicine. 2014; 10:48.

13. Parthiban R, Vijayakumar S, Prabhu S, Yabesh JGEM. Quantitative traditional knowledge of medicinal plants used to treat livestock diseases from Kudavasal taluk of Thiruvarur district, Tamil Nadu, India. Revista Brasileira de Farmacognosia. 2016;26:109-21.

14. Tantengco OAG, Condes MLC, Estadilla HHT, Ragragio EM. Ethnobotanical survey of medicinal plants used by ayta communities in dinalupihan, bataan, Philippines. Pharmacog J. 2018;10(5):859-70.

15. Asase A, Akwetey GA, Achel DG. Ethnopharmacological use of herbal remedies for the treatment of malaria in the Dangme West District of Ghana. J Ethnopharmacol. 2010;129:367-76.

16. Malan DF, Neuba DFR, Kouakou KL. Medicinal plants and traditional healing practices in ehotile people, around the aby lagoon (eastern littoral of Côte d'Ivoire). Journal of Ethnobiology and Ethnomedicine. 2015;11:21.

17. Tahraoui A, El-Hilaly J, Israili ZH, Lyoussi B. Ethnopharmacological survey of plants used in the traditional treatment of hypertension and diabetes in southeastern Morocco (Errachidia province). J Ethnopharmacol. 2007;110:105-17.

18. Fakchich J, Elachouri M. Ethnobotanical survey of medicinal plants used by people in Oriental Morocco to manage various ailments. J Ethnopharmacol. 2014; 154:76-87.

19. Meddour R, Meddour OS, Derridj A. Medicinal plants and their traditional uses in Kabylia (Algeria): an ethnobotanical survey. Planta Medica. 2011;77:PF29.

20. Azzi R, Djaziri R, Lahfa F, Sekkal FZ, Benmehdi H, Belkacem N. Ethnopharmacological survey of medicinal plants used in the traditional treatment of diabetes mellitus in the North Western and South Western Algeria. Journal of Medicinal Plants Research. 2012;6:2041-50.

21. Bouzabata A. Traditional treatment of high blood pressure and diabetes in Souk Ahras district. Journal of Pharmacology and Phytotherapy. 2013;5:12-20.

22. Benarba B, Meddah B. Ethnobotanical study, antifungal activity, phytochemical screening and total phenolic content of Algerian Aristolochia longa. J Intercultural Ethnopharmacol. 2014;3(4):150-4.

23. Benarba B, Belabid L, Righi K, Bekkar A, Elouissi M, Khaldi A, et al Ethnobotanical study of medicinal plants used by traditional healers in Mascara (North West of Algeria). J Ethnopharmacol. 2015;175:626-37.

24. Chermat S, Gharzouli R. Ethnobotanical study of medicinal flora in the North East of Algeria: An empirical knowledge in djebel zdimm (setif). Journal of Materials Science and Engineering. 2015;A5(1-2):50-9.

25. Bakiri N, Bezzi M, Khelifi L, Khelifi-Slaoui M. Ethnobotanical survey of a medicinal plant Peganum harmala L. in the region of M'Sila. Journal Agriculture. 2016;1:38-42.

26. Telli A, Esnault MA, Ould El Hadj Khelil A. An ethnopharmacological survey of plants used in traditional diabetes treatment in south-eastern Algeria (Ouargla province). Journal of Arid Environments. 2016;127:82-92.

27. Boughrara B, Legseir B. Ethnobotanical study close to the population of the extreme north east of Algeria: The municipalities of El Kala National Park (EKNP). Ind Crops Prod. 2016;88:2-7.

28. Ouelbani R, Bensari S, Mouas TN, Khelifi D. Ethnobotanical investigations on plants used in folk medicine in the regions of Constantine and Mila (Northeast of Algeria). Journal of Ethnopharmacology. 2016;194:196-218.

29. Heywood VH. Ethnopharmacology, food production, nutrition and biodiversity conservation: towards a sustainable future for indigenous peoples. Journal of Ethnopharmacology. 2011;137:1-15.

30. Cakilcioglu U, Turkoglu I. Anethnobotanical survey of medicinal plants in Sivrice (Elazı g-Turkey). Journal of Ethnopharmacology. 2010;132:165-175.

31. Ugur C, Selima K, Ismail T, Sukru H. Ethnopharmacological survey of medicinal plants in Maden (Elazig-Turkey). J Ethnopharmacol. 2011;137:469-486.

32. Ababou A, Chouieb M, Bouthiba A, Saidi D, Mederbal K. Floristic diversity patterns in the Beni-Haoua Forest (Chlef, Algeria). Ecologia mediterranea. 2015;41(2):73-84.

33. Senouci F, Ababou A, Chouieb M, Amrous N, Boukeffoussa Ghoul NH. Study of the floristic diversity in Bissa forest, Chlef, Algeria. Algerian Journal of Research and Technology. 2017;02(01):11-9.

34. Akerreta S, Cavero RY, Calvo M I. First comprehensive contribution to medical ethnobotany of Western Pyrenees. Journal of Ethnobiology and Ethnomedicine. $2007 ; 3: 1-26$ 
35. Quezel P, Santa S. New flora of Algeria and southern desert regions, Tome I and Tome II. Paris : CNRS;1962-63;1087.

36. Dobignard A, Chatelain C. Synonymic Index of North African Flora (4 vol.). Geneva: C.J.B.G;2010-13.

37. Morvin Yabesh JE, Prabhu S, Vijayakumar S. An ethnobotanical study of medicinal plants used by traditional healers in silent valley of Kerala, India. Journal of Ethnopharmacology. 2014;154:774-789.

38. Mehdioui $R$, kahouadji A. Ethnobotanical study among the riparian population of the Amsittene forest: case of the Municipality of Imi'Tlit (Province of Essaouira). Bulletin of the Scientific Institute, Rabat, Section Life Sciences. 2007;29:11-20.

39. Benkhnigue O, Zidane L, Mohamed F, Elyacoubih RA, Douira A. Ethnobotanical study of medicinal plants in the region of Mechraâ Bel Ksiri (Gharb Region of Morocco). Acta Bot Barcinonensis. 2010;53:191-216.

40. Sargin SA, Selvi S, Büyükcengiz M. Ethnomedicinal plants of Aydincik District of Mersin, Turkey. J Ethnopharmacol. 2015;174:200-216.

41. Alaoui A, Laaribya S, Gmira N, Benchekroun F. The role of women in local development and the preservation of forest resources cases of the municipality of Sehoul in Morocco. Review of the Mediterranean forest. 2012;xxxiii(4):369-78.

42. Alaoui A, Laaribyia S. Ethnobotanical and floristic study in the rural communes of Sehoul and Sidi Abderrazak (case of Maamora-Northern Morocco). Algerian, Nature and Technology Journal. 2017;B(17):15-24

43. Miliauskas G, Venskutonis PR, Van Beek TA. Screening of radical scavenging activity of some medicinal plants and aromatic plant extract. Food Chem. 2004:85:231-7.

44. Khled Khoudjaa N, Boulekbache-Makhlouf L, Madani K. Antioxidant capacity of crude extracts and their solvent fractions of selected Algerian Lamiaceae. $J$ Industrial Crops and Products. 2014;52:177-82.

45. Maulidiani Abas F, Khatib A, Shaari K, Laijs NH. Chemical characterization and antioxidant activity of three medicinal Apiaceae species. J Industrial Crops and Products. 2014;55:238-47.

46. Rejdali M. The Morocco Flora: Current state and conservation prospects. Biological Diversity and Valorisation of Medicinal Plants. Actes ed. 1996:17-22.

47. González-Tejero MR, Casares-Porcel M, Sánchez-Rojas $C P$, Ramiro-Gutiérrez JM, Molero-Mesa J, Pieroni A, et al. Medicinal plants in the mediterranean area: Synthesis of the results of the project Rubia. J Ethnopharmacol. 2008;116:341 57.

48. Ziyyat A, Legssyer A, Mekhfi H, Dassouli A, Serhrouchni M, Benjelloun W. Phytotherapy of hypertension and diabetes in oriental Morocco. Journal of Ethnopharmacology. 1997;58(1):45-54

49. El Moussaouiti M, Talbaoui A, Gmouh S, Aberchane M, Benjouad A, Bakri $Y$, et al. Chemical composition and bactericidal evaluation of essential oil of Tetraclinis articulata burl wood from Morocco. Journal of the Indian Academy of Wood Science. 2010;7(1-2):14-8

50. Bourkhiss M, Lakhlifi T, Chaouch A, Ouhssine M. Interests of essential oil of Berberie thuya. Phytotherapy. 2015;14(2):109-11.

51. Talbaoui A, El Hamdaoui L, El Moussaouiti M, Aneb M, Amzazi S, Bakri Y. GCMS analysis and antibacterial activity of hydro-distillation oil from Tetraclinis articulata wood grown in Khemisset (Morocco). Journal of the Indian Academy of Wood Science. 2016;13(2):114-7.

52. Longo L, Scardino A, Vasapollo G. Identification and quantification of anthocyanins in the berries of Pistacia lentiscus L., Phillyrea latifolia L. and Rubia peregrina L. Innovative Food Science and Emerging Technologies. 2007;8(3):360-4

53. Dahmoune F, Spigno G, Moussi K, Remini H, Cherbal A, Madani K. Pistacia lentiscus leaves as a source of phenolic compounds: Microwave-assisted extraction optimized and compared with ultrasound-assisted and conventional solvent extraction. Industrial Crops and Products. 2014;61:31-40.

54. Ansari SH, Nahida SH, Siddiqui AN. Pistacia lentiscus: A review on phytochemistry and pharmacological properties. Int J Pharm Pharm Sci. 2012;4(4):16-20.

55. Barra A, Coroneo V, Dessi S, Cabras P, Angioni A. Characterization of the volatile constituents in the essential oil of Pistacia lentiscus L. from different origins and its antifungal and antioxidant activity. J Agric Food Chem. 2007;55(17):7093-8.

56. Eddouks M, Ouahidi ML, Farid O, Moufid A, Khalidi A, Lemhadri A. The use of medicinal plants in the treatment of diabetes in Morocco. Phytother 2007:5(4):194-203.
57. Hyun TK, Kim HC, Kim JS. Antioxidant and antidiabetic activity of Thymus quinquecostatus Celak. Ind Crops Prod. 2014;52:611-6.

58. Ben Arfa A, Combes S, Preziosi-Belloy L, Gontard N, Chalier P. Antimicrobia activity of carvacrol related to its chemical structure. Lett Appl. Microbiol. 2006;43:149-54

59. Shukla S, Gupta S. Apigenin: A promising molecule for cancer prevention Pharma Res. 2010;27(6):962-78

60. Venturini ME, Blanco D, Oria R. In vitro antifungal activity of several antimicrobial compounds against Penicillium expansum. J food Prot. 2012;65(5):834-9.

61. Fachini-Queiroz FC, Dalva de Barros Carvalho M, Kummer R, Cunha JM, Estevao-Silva CF, Grespan R et al. Effects of thymol and carvacrol, constituents of thymus vulgaris I. essential oil, on the inflammatory response. J Evid Based Complementary and Altern Med. 2012;657026:1-10.

62. Miara MD, Ait Hammou M, Hadjadj-Aoul S. Phytotherapy and taxonomy of spontaneous medicinal plants in the region of Tiaret (Algeria). Phytotherapy. 2013;11:206-18.

63. Sari M, Sarri D, Hendel N, Boudjelal A Ethnobotanical study of therapeutic plants used to treat arterial hypertension in the hodna region of Algeria. Global Journal of Research on Medicinal Plants and Indigene Medicine. 2012;1:411-7.

64. Hammiche V, Maiza K. Traditional medicine in Central Sahara: Pharmacopeia of Tassili N'ajjer. J Ethnopharmacol. 2006;105:358-67.

65. Sarri M, Mouyeta FZ, Benzianea M, Cherieta A. Traditional use of medicinal plants in a city at steppic character (M'sila, Algeria). Journal Pharmacy and Pharmacognosy Research. 2014;2:31-5.

66. Rebbas K, Bounar R, Gharzouli R, Ramdani M, Djellouli Y, Alatou D. Plants of medicinal and ecological interest in the region of Ouanougha (M'Sila, Algeria). Phytotherapy. 2012;10:131-42

67. Parada M, Carrió E, Bonet MA, Vallès J. Ethnobotany of the Alt Empordà region (Catalonia, Iberian Peninsula) Plants used in human traditional medicine. $J$ Ethnopharmacol. 2009;124:609-18.

68. Tuttolomondo A, Pecoraro R, Pinto A. Studies of selective TNF inhibitors in the treatment of brain injury from stroke and trauma: a review of the evidence to date. Drug Des Devel Ther. 2014;8:2221-38.

69. Guzel F, Saygili H, Gulbahar AS, Filiz K. New low-cost nanoporous carbonaceous adsorbent developed from carob (Ceratonia siliqua) processing industry waste for the adsorption of anionic textile dye: characterization, equilibrium and kinetic modeling. J Mol Liq. 2015;206:244-55.

70. Yemele MD, Telefo PB, Lienou LL, Tagne SR, Fodouop CSP, Goka CS, et al. Ethnobotanical survey of medicinal plants used for pregnant women's health conditions in Menoua division-West Cameroon. J Ethnopharmacol. 2015;160:14-31.

71. Salhi S, Fadli M. Medicinal plants of the city of Kenitra (Morocco). J Lazaroa 2006;31:131-46.

72. Raterta R, de Guzman GQ, Alejandro JGD. Assessment, inventory and ethnobotanical survey of medicinal plants in Batan and Sabtang Island (Batanes Group of Islands, Philippines). International Journal of Pure and Applied Biosciences. 2014;2:147-54

73. Francis Xavier $T$, Kannan M, Auxilia A. Observation on the traditiona phytotherapy among the Malayali tribes in Eastern Ghats of Tamil Nadu, South India. Journal of Ethnopharmacology. 2015;165:198-214.

74. Benitez G, Gonzalez Tejero MR, Molero-Mesa J. Pharmaceutical ethnobotany in the western part of Granada province (southern Spain): Ethnopharmacological synthesis. J Ethnopharmacol. 2010;129:87-105

75. Daoudi A, Bammou M, Zarkani S, Slimani I, Ibijbijen J, Nassiri L. Ethnobotanical study of medicinal flora in the rural municipality of Aguelmouss province of Khenifra (Morocco). Phytotherapy. 2016;14(4):220-8.

76. Slimani M, Najem R, Belaidi L, Bachiri EH, Bouiamrine L, Nassiri Ibijbijen J. Ethnobotanical Survey of medicinal plants used in Zerhoun region Morocco. IJIAS. 2016;15:846-63.

77. Paksoy MY, Selvi S, Savran A. Ethnopharmacological survey of medicinal in Ulukis, la (Nigde-Turkey). J Herb Med. 2016:1-7.

78. Punnam Chander M, Kartick C, Gangadhar J, Vijayachari P. Ethno medicine and healthcare practices among Nicobarese of Car Nicobar - An indigenous tribe of Andaman and Nicobar slands. Journal of Ethnopharmacology. 2014:158:18-24.

79. Azaizeh H, Saad B, Khaleel K, Said O. The state of the art of traditional Arab herbal medicine in the eastern region of the Mediterranean: A review. eCAM. 2006;3:229-35 
80. Ben Ammar R, Ben Sghaier M, Boubaker J, Bhouri W, Bouhlel I, Kilani S, et al. Antioxidant activity and inhibition of aflatoxin B1-,nifuroxazide- and sodium azide-induced mutagenicity by extracts from Rhamnus alaternus L. ChemicoBiological Interactions. 2008;174:1-10
81. Khacheba I, Djeridane A, Yousfi M. Twenty traditional Algerian plants used in diabetes therapy as strong inhibitors of and-amylase activity. International Journal of Carbohydrate Chemistry. 2014:1-12

\section{GRAPHICAL ABSTRACT}

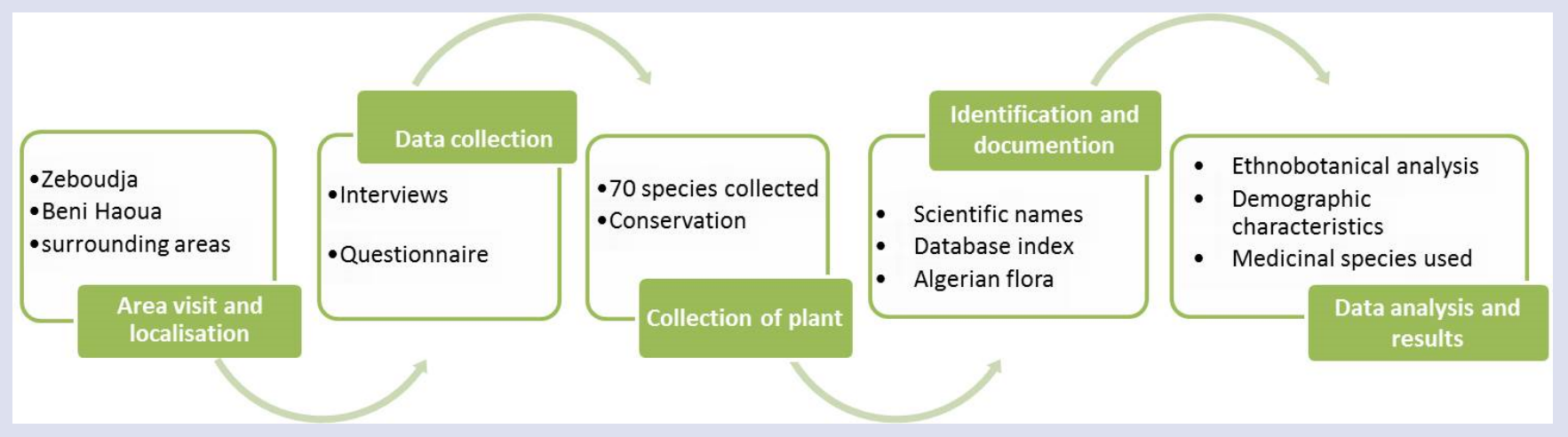

\section{SUMMARY}

This paper documented the results of ethnobotanical study carried out in the northeastern Dahra Mountains, Algeria. A total of 70 species belonging to 38 families. Lamiaceae and Asteraceae were the most used plant family by the local population. Leaves were the most used part, the majority of remedies were prepared as a decoction and gastro-intestinal system disorders were the most treated diseases. This first ethnobotanical study conducted in the region of Bissa revealed a very rich local knowledge in term of traditional herbal medicine.

\section{ABOUT AUTHORS}

Fatima Senouci: Assistant Professor, Department of Water Environment and Sustainable Development, Faculty of Nature and Life Sciences, University Hassiba Ben Bouali, Chlef. PhD student, Department of Biology, Faculty of Nature and Life Sciences, University Abd El Hamid Ibn Badis, Mostaganem, Algeria.

Prof. Dr. Adda Ababou: Department of Biology, Faculty of Nature and Life Sciences, University Hassiba Ben Bouali, Chlef, Algeria.

Dr. Mohammed Chouieb: Department of Agronomy, Faculty of Nature and Life Sciences, University Abd El Hamid Ibn Badis, Mostaganem, Algeria.

Cite this article: Senouci F, Ababou A, Chouieb M. Ethnobotanical Survey of the Medicinal Plants used in the Southern Mediterranean. Case Study: The Region of Bissa (Northeastern Dahra Mountains, Algeria). Pharmacog J. 2019;11(4):647-59. 